for the person with epilepsy, but these findings also show that poor adjustment is not inevitable.

JE CHAPLIN

Rehabilitation Resource Centre, Department of Systems Science, City University, Northampton Square, London ECIVOHB, UK

INSEG (Institute of Neurology and National Society for Epilepsy Research Group), Institute of Neurology, London and Chalfont Centre for Epilepsy

Rehabilitation Resource Centre R YEPource Centre INSEG, Institute of Neurology

Correspondence to: Dr Chaplin

1 Chaplin JE, Yepez Lasso R, Shorvon S, Floyd $M$. A quantitative approach to measuring the social effects of epilepsy. Neuroepidemiology 1990;9:151-8.

2 Chaplin JE, Yepez Lasso R, Shorvon SD, Floyd $M$. National general practice study of epilepsy: the social and psychological effects of a recent diagnosis of epilepsy. BMF 1992;304:1416-8.

3 Hart Y, Sander J, Shorvon S. National general practice study of epilepsy and epileptic practice study of eplepsy and epileptic seizures: objectives and study methodology ported prospective cohort study of epilepsy. Neuroepidemiology 1989;

4 Hart Y, Sander J, Johnson A, Shorvon S. National general practice study of epilepsy: recurrence after a first seizure. Lancet 1990;336:1271-4.

5 Sander J, Hart Y, Johnson A, Shorvon S. National general practice study of epilepsy: newly diagnosed epileptic seizures in a general population. Lancet 1990;336:

Spinal somatosensory potential monitoring in three cases of neurological deterioration after laminectomy for cervical spondylotic myelopathy

Posterior cervical laminectomy is a widely accepted treatment for multisegmental cervical spondylotic myelopathy, particularly when the condition is associated with a narrow spinal canal. ${ }^{1}$ After laminectomy, some patients complain of increased weakness and paraesthesiae of the arms. This clinical finding is consistent with segmental damage either at the spinal cord or nerve root level. We report three patients of transient neurological deterioration in whom spinal somatosensory potential monitoring during operation gave useful information on the pathophysiology of the postoperative deficit.

Cervical spinal somatosensory potentials were recorded during operation, before and after laminectomy. The potentials were evoked by electrical stimulation of the median nerve at the wrist. Rectangular pulses $(0.5 \mathrm{~ms}$ duration and amplitude $4 / 3$ of the motor threshold) were delivered at 1 cycle/s. After exposure of the cervical laminae, the recording electrode (Medtronic Sigma 3483 or Quad 3487A) was placed in the epidural space, medially over the posterior columns. The reference electrode consisted of a $14 \mathrm{G}$ needle inserted into the paraspinal muscles immediately caudal to the skin incision. A total of 50-150 stimuli were applied and averaged; analysis time was $50 \mathrm{~ms}$ with a horizontal resolution of 98 $\mu$ s per point. An open bandpass (2-5000 $\mathrm{Hz}$ ) was set and the negative upward convention was used. The potentials were analysed for latency, amplitude, duration, and waveform. The single components were labelled according to their polarity and to the expected latency.

Anaesthesia was induced with thiopentone sodium $(5 \mathrm{mg} / \mathrm{kg})$ and fentanyl $(0.002$
Case $1:$ C3-C7 posterior laminectomy

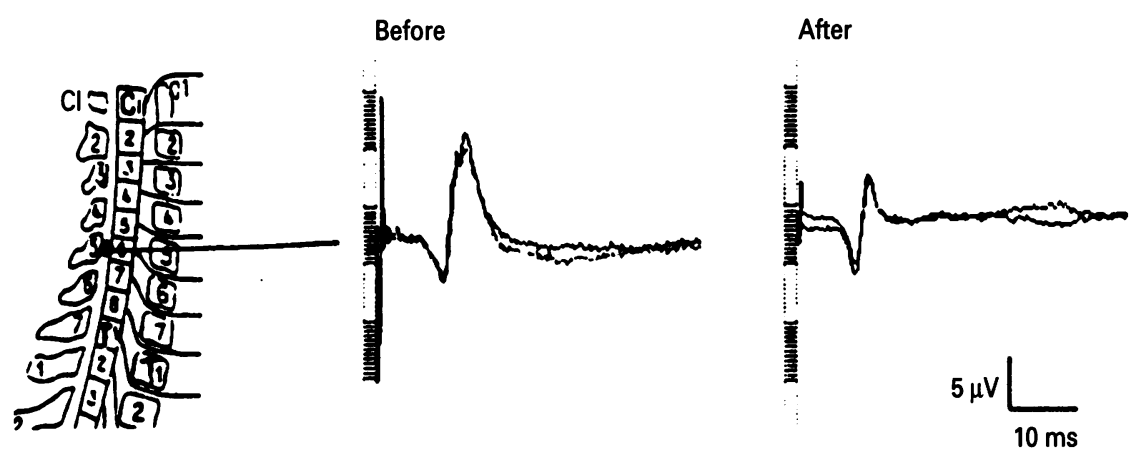

Case 2 : C3-C5 posterior laminectomy

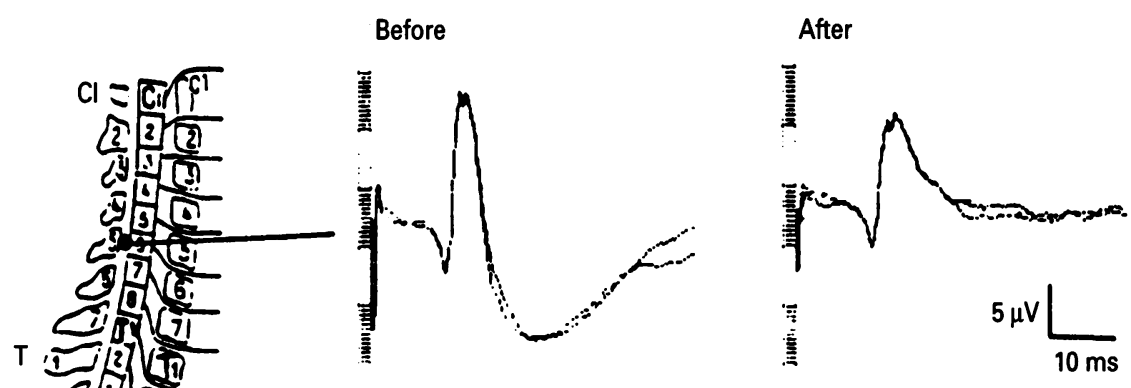

Case 3 : $\mathrm{C} 3-\mathrm{C} 7$ posterior laminectomy

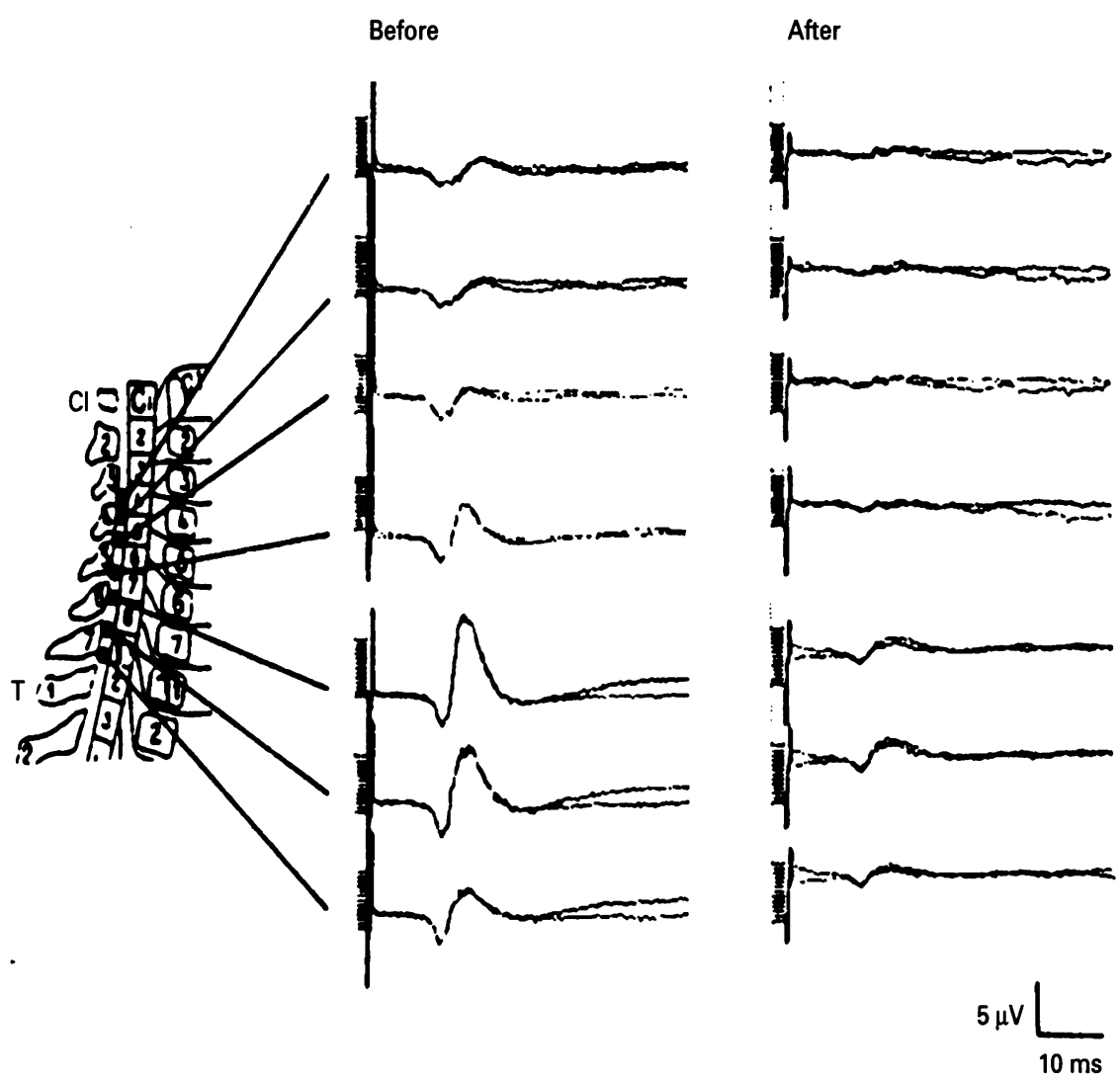

Cervical epidural somatosensory potentials evoked by median nerve stimulation at the wrist $(0.5 \mathrm{~ms}, 1 \mathrm{c} / \mathrm{s}, 4 / 3$ of motor threshold) recorded before and after laminectomy for cervical spondylotic myelopathy. 
$\mathrm{mg} / \mathrm{kg}$ ). Endotracheal intubation was performed during muscle relaxation with pancuronium $(0.1 \mathrm{mg} / \mathrm{kg})$. Anaesthesia was maintained with nitrous oxide, oxygen, and isoflurane $(0 \cdot 7-1 \cdot 2 \%)$.

Case 1 was a 51 year old man who, since 1986, had had a progressive spastic paraparesis and who complained of cervicobrachial pain. Neuroradiological investigations showed cervical spondylosis with a narrow canal from $\mathrm{C} 3$ to $\mathrm{C} 7$. The patient underwent a $\mathrm{C} 3-\mathrm{C} 7$ posterior laminectomy. After operation he complained of weakness and paraesthesiae in both hands. Motor and sensory functions in the arms were restored after three to four weeks.

Before laminectomy, the potential recorded epidurally at $\mathrm{Cv} 5$ after median nerve stimulation was characterised by a normal P10 followed by an N11 wave with a peak latency of $12.3 \mathrm{~ms}$, and an N13 wave at $13.9 \mathrm{~ms}$. Amplitude (P10-N13: 12.8 $\mu \mathrm{v}$ ) and waveform were normal. After laminectomy, the disappearance of the $\mathrm{N} 13$ wave was noticed. The P10 and N11 waves were unchanged (figure).

Case 2 was a 56 year old man who presented in 1984 with progressive spastic tetraparesis and hypoaesthesia below $\mathrm{C4}$. An MRI of the cervical spine showed spondylotic stenosis of the canal from $\mathrm{C} 3$ to C5. He underwent a C3-C5 posterior laminectomy. After operation, he showed a transient (seven days) decrease of motor performance in the distal segments of the arms.

Before laminectomy, the potential evoked by median nerve stimulation at $\mathrm{Cv} 5$ consisted of a slightly delayed P10 (peak latency: $11.2 \mathrm{~ms}$ ), N11 (peak latency: $13.6 \mathrm{~ms}$ ), N13 (latency: $14.9 \mathrm{~ms}$ ), followed by a slow positive wave (P18 at $25.8 \mathrm{~ms}$ ). Amplitude (P10-N13: $15 \mu \mathrm{v})$ and waveform were normal. After laminectomy a decreased amplitude (P10-N13: $10.7 \mu \mathrm{v})$ and a longer duration $(11.8 \mathrm{~ms} v$ a prelaminectomy value of $4 \mathrm{~ms}$ duration) of the main negative wave (N13) were evident (figure).

Case 3 was a 67 year old man with spastic tetraparesis and hypoaesthesia of both hands. Cervical MRI showed a multisegmental spondylosis from $\mathrm{C} 4$ to $\mathrm{C} 7$. The patient underwent a C3-C7 posterior laminectomy. After operation a transient (four weeks) increase of the paresis and hypoaesthesia of upper extremities was evident.

Before laminectomy, the epidural evoked potentials consisted of a slightly delayed P10 (peak latency $11.3 \mathrm{~ms}$ ) followed by a negative wave peaking at $14 \mathrm{~ms}$. The amplitude was normal from Th1 to Cv6 (P10N13: $10 \mu \mathrm{v}$ ), whereas it was decreased above Cv5-6 (P10-N13 at Cv5-6: $5 \mu \mathrm{v})$. After laminectomy a decrease in amplitude or disappearance of the main negative wave (N13) was evident (figure).

In these three cases, laminectomy was followed by an alteration in the postsynaptic component (N13) of the median nerve evoked potential, whereas the root component (N11) was unchanged. ${ }^{2}$ This is consistent with grey matter damage. The genesis of this damage is probably a vascular insult in an already hypoperfused area. ${ }^{34}$ Indeed, in cervical spondylotic myelopathy the osteophytic process may impinge on the anterior spinal artery and cause hypoperfusion with loss of the haemodynamic autoregulation in the spinal cord watershed areas. ${ }^{5}$ The decompression by posterior laminectomy may increase the blood supply, but as a consequence of the previously mentioned loss of haemodynamic autoregulation, it may cause hyperaemia in these areas and induce the paradoxal effect of a further ischaemia. Other mechanisms of damage such as cervical spine instability, a local concussion of the cord, or a temporary oedema caused by mechanical interference, cannot be excluded.

In conclusion, this neurophysiological study shows that neurological deterioration after posterior laminectomy in these patients was due to grey matter damage and not to nerve root stretching. It is important to stress that the damage reversed spontaneously.

B CIONI M MEGLIO L PENTIMALLI Istituto di Neurochirurgia, Università Cattolica,
Roma, Italy

We thank Anna Capuano for her technical assistance in collecting the data. The work was partially supported by MPI and by the V Smith
Foundation for Restorative Neurology, Houston, Texas, USA.

Correspondence to: Dr Beatrice Cioni, Istituto di Neurochirurgia, Università Cattolica, Lg A Gemelli 8, 00168 Roma, Italy.

1 Yonenobu K, Takeshi F, Keiro O, Okada K, Yamamoto T, Harada N. Choice of surgical treatment for multisegmental cervical spondylotic myelopathy. Spine 1985;10: $710-6$.

2 Cioni B, Meglio M. Epidural recordings of electrical events produced in the spinal cord by segmental, ascending and descending volleys. Applied Neurophysiology 1989;49: 315-26.

3 Cybulski G, D'Angelo C. Neurological deterioration after laminectomy for spondylotic cervical myeloradiculopathy: the putative Neurosurg Psychiatry 1988;51:717-8.

4 Levy WJ, Dohn DF, Hardy RW. Central cord syndrome as a delayed postoperative comsyndrome as a delayed postoperative com-
plication of decompressive laminectomy. plication of decompressive

5 Hoff J, Nishimura M, Pitts L, Vilnis V, Tuerk $K$, Lagger $R$. The role of ischemia in the $\mathrm{K}$, Lagger R. The role of ischemia in the pathogenesis of cervical spol
myelopathy. Spine 1977;2:100-8.

\section{Different criteria in the assessment of} visuospatial neglect

The classic manifestations of visuospatial neglect include (1) a certain minimal number of omissions in conventional neglect tests, and (2) a defective performance more pronounced at the side opposite the brain lesion. ${ }^{2}$ The procedure for assigning patients to the spatial neglect group typically includes a measure of the number of omissions in tests such as target cancellation, figure copying, and freehand drawing. A measure of the asymmetry of omissions is, however, not always included as a criterion for spatial neglect. ${ }^{3-5}$ Unless this is done, patients showing the typical pattern of visuospatial neglect may be mixed with patients showing no laterality in the defective performance.

The aim of this study was to compare the accuracy of the assignment of patients to the neglect group when using three different criteria for visuospatial neglect. A consecutive series of 60 right handed patients, mean (SD) age $60.25(12.58)$; range $21-77$ years with a single right hemispheric stroke were assessed one to eight weeks after stroke (mean (SD) 2.5 (1.53) weeks) using seven subtests from a shortened and slightly modified version of the behavioural inattention test. (The behavioural inattention test was introduced by Wilson et al to offer a standardised test battery of unilateral visual neglect. ${ }^{3}$ ) Thirty four neurological healthy age matched right handed subjects served as controls.

The cut off level for defective numbers of omissions in each subtest and in the total number of subtests was represented by the first score below the normative range obtained from the control group. The asymmetry of defective performance was measured in the tests with scores at or below the cut off level. To avoid confusion of centrally and laterally located omissions, only the lateral parts of the test material were included in this measurement. ${ }^{6}$ The number of detected targets at the contralesional side was divided by the total number of detected targets ${ }^{7}$ and then represented by a percentage measure. The classification of the asymmetry of omissions was based on the range of laterality scores found in the control group (44-56\%). It was classified as contralesional asymmetry if a laterality score below $44 \%$ was found in at least one of the tests and if no test was found with a score above $56 \%$.

An assessment of hemianopia (by standard confrontational techniques) was included to analyse the influence of hemianopia on the asymmetric performance. Contralesional asymmetry as well as hemianopia were exclusively found in patients with neurological deficits remaining three weeks after stroke (in patients showing a major stroke, $n=41$ ). No association between the presence of hemianopia $(n=15)$ and contralesional asymmetry $(n=18)$ was found in this group $\left(\chi^{2}=2 \cdot 49, p=0 \cdot 115\right)$.

In the present study, we compared three different criteria for assigning patients to the visuospatial neglect group, which are similar to criteria applied in recent studies of visual neglect ${ }^{478}$ : (1) a score at or below the individual cut off score in any of the seven subtests; (2) a total score at or below the aggregate cut off score for the seven subtests in the battery; (3) a score at or below the cut off level in at least one of the subtests and a contralesional asymmetry in the defective performance (as defined earlier). A significant difference was obtained between the proportions of patients defined as having visuospatial neglect when using the three criteria for neglect $(Q=19, \mathrm{df}=$ $2, p<0.001$, Cochran $Q$ test). The proportions were $45 \%(27 / 60), 23 \%(14 / 60)$, and $30 \%(18 / 60)$ respectively.

The criterion of individual cut off scores was the most generous method for classifying patients as having neglect. The use of this criterion resulted in nine patients (Nos $12,15,19-21$, and 24-27) who were misidentified as having visuospatial neglect, although no contralesional asymmetry was found in the defective tests (table). These results show that visual inattention in a conventional test battery of neglect might be present with no contralesional asymmetry in the inattentive behaviour. The findings emphasise the importance of including not only visual inattention as a criterion for visual neglect, but also the asymmetry of the inattentive behaviour.

The aggregate cut off criterion was the most restrictive method for classifying patients as having neglect. Thus the table shows that five patients (Nos 16-18,22, and 23) were classified as non-neglect patients, although the traditional neglect pattern with a defective number of omissions and a 